\title{
Letter to the Editor: Ascending Pharyngeal Collaterals Between the External Carotid Artery and the Occluded Internal Carotid Artery
}

\section{To the editor:}

I have read with a great interest the article written by Takeuchi et al.7) They described a vidian artery as an important collateral between the external carotid artery and the occluded internal carotid artery (ICA). Understanding of the collateral pathways in the case of an occlusion or a stenosis of the cervical ICA is clinically of utmost importance because of the expanding therapeutic options today, including medication, bypass surgery, carotid endarterectomy, and carotid stenting. Neuroradiological examination in such cases is crucial and an embryological knowledge of the cerebral vessels is required for its proper interpretation. ${ }^{2)}$

The vidian artery is a remnant of the first aortic arch (mandibular arch) which passes through the pterygoid canal (also known as the vidian canal) from the pterygopalatine fossa posteriorly to the extracranial portion of the foramen lacerum, where it anastomoses with a mandibular artery of the ICA. ${ }^{5)}$ On the lateral angiogram, this vidian artery runs at the skull base horizontally. Although Takeuchi et al. described a vidian artery as a collateral to the distal ICA, judging from the three lateral angiograms provided in their paper, no vidian artery was demonstrated. Instead, a large ascending pharyngeal artery (APA) was shown, which was apparently the main collateral from the external carotid artery to the distal ICA without a vidian collateral. They explained that the anastomosis was between the APA and vidian artery through the nasopharyngeal arterial network, but the location of the APA anastomosis was not in the nasopharynx, but in the petrous bone. I believe that there are two possibilities for the angio-architecture in their case. One is that the inferior tympanic branch of the APA directly supplies the distal ICA. The tympanic branch penetrates Jacobson's canal at the skull base between the carotid and jugular foramina, enters the tympanic cavity, and anastomoses with the carotico-tympanic artery (a remnant of the second aortic arch: hyoid artery) through the tympanic arterial plexus. ${ }^{4)}$ The second possibility is a collateral of the APA to the mandibular artery using dural branches through the foramina ovale, Vesalius (sphenoidal emissary foramen), or lacerum. ${ }^{6}$ ) The straight course of the APA branch (possibly the inferior tympanic branch) in addition to the anteriorly located pharyngeal branch in their Fig. 2 and a low-set anastomotic point in the petrous bone suggest the former explanation is most likely.
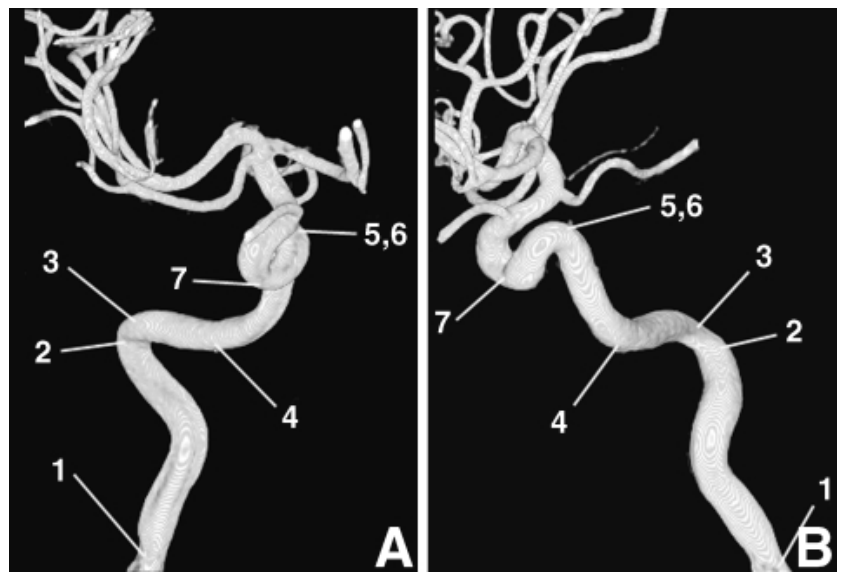

Fig. 1 Possible collaterals of the internal carotid artery. A: anteroposterior view, B: lateral view. The numbers indicate the possible anastomotic points of the collaterals: 1, hypoglossal artery; 2, otic artery; 3, carotico-tympanic artery; 4, mandibular artery; 5, trigeminal artery; 6, maxillary artery; and 7 , inferolateral trunk.

Embryologically, there are several possible points of collaterals on the ICA between the cervical carotid bifurcation and the ophthalmic artery, i.e., hypoglossal artery, otic artery, hyoid artery (carotico-tympanic artery), mandibular artery, trigeminal artery, maxillary artery, and dorsal ophthalmic artery (inferolateral trunk) in caudocranial order (Fig. 1). ${ }^{3)}$ If the collateral is in the petrous bone, it must be either the caroticotympanic artery or mandibular artery because the otic artery is a theoretical artery that may not exist. ${ }^{1)}$ To differentiate the former two, the anteroposterior view of the angiogram is useful since the mandibular artery is located near the medial turn of the petrous ICA whereas the carotico-tympanic artery is located near the apical turn at the carotid genu.

In summary, the collateral in Takeuchi's report seems not to use the remnant of the first aortic arch (vidian artery), but uses the remnants of the third aortic arch (APA) and the second aortic arch (carotico-tympanic artery).

Masaki KomiYama, M.D. Department of Neurosurgery Osaka City General Hospital Osaka, Japan 


\section{References}

1) Bhattacharya JJ, Lamin S, Thammaroj J: Otic or mythic? AJNR Am J Neuroradiol 25: 160-161, 2004

2) Komiyama $M$ : [Functional anatomy of the cerebral arteries]. No Shinkei Geka 33: 213-224, 2005 (Jpn)

3) Lasjaunias PL: Segmental identity and vulnerability in cerebral arteries. Interventional Neuroradiology 6: 113-124, 2000

4) Lasjaunias P, Moret J: The ascending pharyngeal artery: normal and pathological radioanatomy. Neuroradiology 11: 77-82, 1976

5) Osborn AG: The vidian artery: normal and pathologic anatomy. Radiology 136: 373-378, 1980

6) Quisling RG, Seeger JF: Ascending pharyngeal artery collateral circulation simulating internal carotid artery hypoplasia. Neuroradiology 18: 277-280, 1979

7) Takeuchi M, Kuwayama N, Kubo M, Umemura K, Hirashima Y, Endo S: Vidian artery as a collateral channel between the external and occluded internal carotid arteries. Case report. Neurol Med Chir (Tokyo) 45: 470-471, 2005

\section{Article under discussion}

Neurol Med Chir (Tokyo) 45 (9), 470 471, 2005

\section{Vidian Artery as a Collateral Channel Between the External and Occluded Internal Carotid Arteries -Case Report-}

Mikinobu TAKEUCHI, Naoya KUWAYAMA, Michiya KUBO, Kimiko UMEMURA, Yutaka HIRASHIMA, and Shunro ENDO

Department of Neurosurgery, Toyama Medical and Pharmaceutical University, Toyama

Abstract

A 68-year-old man presented with occlusion of the internal carotid artery (ICA) manifesting as a 6-month history of progressive sensory and motor disturbance of the left lower limb. Angiography clearly demonstrated a collateral arterial network between the ICA and external carotid artery (ECA) through the vidian artery, a small branch of both the ICA and ECA. The vidian artery may form an unusual but important ECA-ICA collateral pathway in patients with occlusive lesion of the ICA. 